\title{
A Retrospective Clinical \& Epidemiological Study on Rabies at A Tertiary Care Infectious Diseases Hospital
}

\author{
Dr.A. Madhav ${ }^{1}$, M.D., Dr.Sunitha ${ }^{2}$, M.D., Dr.Meera M³ ${ }^{3}$ M.D., \\ ${ }^{1}$ Assistant Professor Of Medicine, Govt. Fever Hospital, Hyderabad, Telangana State, India \\ ${ }^{2}$ Civil Assistant Surgeon, Govt. Fever Hospital, Hyderabad, Telangana State, India \\ ${ }^{3}$ Professor Of Microbiology, Govt. Fever Hospital, Hyderabad, Telangana State, India
}

\begin{abstract}
Human rabies, transmitted through the bite of a rabid animal, is a preventable viral disease of CNS associated with $100 \%$ mortality. The present study was done at Sir Ronald Ross Institute of Tropical and Communicable Diseases (SRRIT\&CD) to assess the burden of the disease and correlate factors like the age, gender, occupation, anatomical site \& nature of bite, type of animal, range of clinical symptoms and immunization status with onset and outcome of the disease which, eventually at present, is death. Ninety eight patients with clinical diagnosis of rabies were admitted from Jan 2011 to Dec 2015. Most affected were male children $<10$ yrs old. Most of the affected were urban / semi urban and semi literate who presented with H/o dog bites on lower limbs and class III in nature. The other animals included cats, wolf and mongoose. While most were stray dogs, some were pets. Hydrophobia and aerophobia were observed to be the commonest presenting features. Most of the patients died in $<24$ hrs. Active immunization in the form of anti rabies vaccine $(A R V)$ was taken by $30 \%$ of patients while majority of the patients failed to wash the wound or receive rabies immunoglobulins $(R I G)$.
\end{abstract}

Keywords: Dog bite, Epidemiology, Rabies, Hydrophobia, Post exposure prophylaxis

\section{Introduction}

Rabies is a $100 \%$ fatal, infectious viral disease of the central nervous system that can primarily affect almost all mammals ${ }^{1}$. In up to $99 \%$ of human cases, rabies virus is transmitted by affected rabied dogs few days before the death of the animal. The infection usually spreads through bites or scratches, when the virus in the saliva of the infected animal gets transmitted to the patient ${ }^{2}$.

Patients who die of rabies are usually those who either do not seek or receive timely treatment. Earliest post exposure prophylaxis in the form of ARV, thorough wound washing \& timely RIG infiltration can be life saving ${ }^{3}$.

\section{Aims \& Objectives:}

1. To assess the disease burden due to rabies

2. To identify the factors associated with animal bite risk in the community-provoked/unprovoked/ seasonal behavior/ appearance of dog/ laboratory or occupational exposure

3. To study variations in clinical presentation of the disease

4. To stress upon the importance of wound management

5. To create awareness on post exposure prophylaxis

6. To stress upon the importance of vaccination of pet animals

\section{Materials \& methods}

A total number of 98 cases from January 2011 to December 2015 with clinical diagnosis of rabies admitted at SRRIT\&CD were included in the present study.

Preliminary data including age, sex, address and occupation were noted. Animal bite and rabies related data was recorded which included time and site of bite, wound irrigation with soap/disinfectant and water, vaccine details and local infiltration with calculated dose of ERIG/HRIG. Information regarding biting animal included stray dog/other mammals, in case of pets, whether vaccinated or not, time since vaccination, silent/furious, provoked/unprovoked, attacked any other person in the community and alive/dead.

Clinical presentation- H/o prodromal illness like fever, vomitings \& myalgia, pain/ local paraesthesia in the form of unexplained or unusual tingling, pricking or burning sensation at the wound site, irritability, restlessness, seizures, photophobia, hyper salivation, breathlessness, altered sensorium, altered behavior, spasms, apart from the well documented hydrophobia and aerophobia were noted for each patient along with duration.

Details of local wound management and vaccines received were noted. 


\section{Results}

The study included retrospective data analysis of 98 cases of rabies admitted from Jan 2011 to Dec 2015 kept under observation at the rabies isolation ward at SRRIT\&CD, Hyderabad. Details of age and gender are shown in Table no. 1.

Table no.1 showing age \& gender details

\begin{tabular}{|l|l|l|l|}
\hline Age & Males & Females & Total \\
\hline $\begin{array}{l}<10 \\
\text { yrs }\end{array}$ & 22 & 05 & 27 \\
\hline $10-$ & 12 & 01 & 13 \\
20 & & & \\
yrs & & 08 & 20 \\
\hline $\begin{array}{l}21- \\
40 \\
\text { yrs }\end{array}$ & 12 & & \\
\hline $\begin{array}{l}41- \\
60 \\
\text { yrs }\end{array}$ & 17 & 16 & 33 \\
\hline $\begin{array}{l}>60 \\
\text { yrs }\end{array}$ & 04 & & \\
\hline Total & 67 & 01 & 05 \\
\hline
\end{tabular}

Patients were from the various districts of Telangana, few were from AP, Maharashtra \& Karnataka and presented with varied clinical symptoms as shown in Table no.2

Table no. 2 showing clinical symptoms of rabies

\begin{tabular}{|l|l|l|}
\hline 1 & Fever, malaise, headache & $05(4.9 \%)$ \\
\hline 2 & Fatigue, anorexia, vomitings & $04(3.9 \%)$ \\
\hline 3 & Paraesthesia & $05(4.9 \%)$ \\
\hline 4 & Anxiety, irritability, restlessness & $10(83.3 \%)$ \\
\hline 5 & Photophobia & $05(4.9 \%)$ \\
\hline 6 & Altered sensorium & $05(4.9 \%)$ \\
\hline 7 & Breathlessness & $14(13.7 \%)$ \\
\hline 8 & Hyper salivation & $06(5.8 \%)$ \\
\hline 9 & Hydrophobia & $98(96 \%)$ \\
\hline 10 & Aerophobia & $90(88 \%)$ \\
\hline 11 & Unconscious & $01(0.9 \%)$ \\
\hline 13 & Spasms & $01(0.9 \%)$ \\
\hline 14 & Seizures & $01(0.9 \%)$ \\
\hline
\end{tabular}

Duration of time from bite to onset of disease varied widely from 5 days to 3 years (Table no. 3 ).

Table No.3 showing interval between bite and onset of disease

\begin{tabular}{|l|l|l|}
\hline S.No & Duration & No. of cases \\
\hline 1 & $5-9$ days & 03 \\
\hline 2 & $10-15$ days & 08 \\
\hline 3 & 15 dayto<1mon & 16 \\
\hline 4 & $1-3$ mon & 45 \\
\hline 5 & $4-6$ mon & 15 \\
\hline 6 & $1-3$ yrs & 06 \\
\hline 7 & No H/o bite & 05 \\
\hline
\end{tabular}

Ninety three patients gave h/o dog bite out of which 2 were due to pet dogs. Cat bites accounted for 3 cases while one male patient aged 30 yrs from Gulbarga sustained wolf bite. There was one mongoose attack involving a 50 year old female from Nalgonda district of Telangana State (Table no. 4).

The sites of the bites included face, legs, arms, thighs, head and fingers (Table no.4).

Table no. 4 showing anatomical site of the bites inflicted by various animals.

\begin{tabular}{|l|l|l|l|}
\hline S.No & Site of bite & Animal & Number \\
\hline 1 & Face & $\begin{array}{l}\text { Stray dog } \\
\text { Wolf }\end{array}$ & 18 \\
& & Stray dog & 01 \\
\hline 2 & Fingers & Stray dog & 03 \\
\hline 3 & Forehead & stray dog & 02 \\
\hline 4 & Head & \multicolumn{2}{|l}{} \\
\hline
\end{tabular}


A Retrospective Clinical \& Epidemiological Study On Rabies At A Tertiary Care ...

\begin{tabular}{|l|l|l|l|}
\hline & & Pet dog & 01 \\
\hline 5 & Leg & Stray dog & 40 \\
\hline 6 & Scratch on leg & Stray dog & 07 \\
\hline 7 & Hand & Pet dog & 01 \\
\hline 8 & Arm and leg & Stray dog & 07 \\
\hline 9 & Thigh & Stray dog & 05 \\
\hline 10 & Scalp & Stray dog & 03 \\
\hline 11 & Leg & Mongoose & 01 \\
\hline 12 & Leg & Cats & 03 \\
\hline 13 & No bite & Puppies & 05 \\
\hline
\end{tabular}

In regards to post exposure prophylaxis received, thirty patients reportedly washed the wound/wounds while all doses of ARV was taken by only 2 and RIG by three (Table no.5)

Table no.5 showing post exposure prophylaxis received

\begin{tabular}{|l|l|l|l|}
\hline S. no & Wound management & $\begin{array}{l}\text { Anti } \\
\text { vaccination }\end{array}$ & $\begin{array}{c}\text { Rabies } \\
\text { Immunoglobulins }\end{array}$ \\
\hline & & 5 doses-02 & Taken-03 \\
\hline 1 & Not washed-39 & 4 doses-07 & Not taken-95 \\
\hline 2 & Washed-30 & 3 doses-09 & \\
\hline 3 & Not recorded-27 & 1 dose-11 & \\
\hline 4 & Washed with herbal medicine-02 & $\begin{array}{l}\text { Full course after 20 } \\
\text { days of bite-01 }\end{array}$ & \\
\hline 5 & & Not taken-68 & \\
\hline
\end{tabular}

All patients died over 1-48 hrs duration post admission (Table no.6)

Table No.6 showing duration from admission till death

\begin{tabular}{|l|l|l|}
\hline S.No & Duration from admission to death & No. of cases \\
\hline 1 & $<1 \mathrm{hr}$ & 01 \\
\hline 2 & $1-2 \mathrm{hrs}$ & 16 \\
\hline 3 & $3-12 \mathrm{hrs}$ & 42 \\
\hline 4 & $13-24 \mathrm{hrs}$ & 27 \\
\hline 5 & $25-48 \mathrm{hrs}$ & 12 \\
\hline & Total & 98 \\
\hline
\end{tabular}

\section{Discussion}

Rabies is a disease of the central nervous system, ultimately invariably leading to death. The early symptoms of rabies in humans usually are general which include fever, headache, general weakness or discomfort, similar to that of many other illnesses. As the disease progresses, more specific symptoms appear and may include insomnia, anxiety, confusion, slight or partial paralysis, excitation, hallucinations, agitation, hyper salivation, difficulty in swallowing, aerophobia, photophobia and hydrophobia. Death usually occurs within days of onset of the symptoms ${ }^{2}$.

The term 'Rabhas' in Sanskrit means 'mad'. In the present study, it has been observed that most of the patients were affected by unprovoked bite of an animal that is already infected with rabies virus and mostly suffering from disease.

The animal involved was stray dog in 91 cases, cat in 3 , pet dog in 2 , single case of wolf bite and an isolated mongoose attack (Table 4).

Seasonal variation in incidence of dog bites was observed during the study, more cases occurring during the pre-monsoon months followed by another shorter peak during post-monsoon. It was also noted that the dog-bites occurred throughout the year ${ }^{4}$ especially in those localities with high population density cohabiting with an equally high number of stray dogs ${ }^{5}$.

Since men are more likely to go out of their homes for work as compared to women and tend to have a casual attitude towards animals, they are found to be more affected as compared to women. Most of the affected men were daily wage workers which exposed them to the risk associated with outdoor activities ${ }^{4}$, stray dog bite included. 
Women are more frequently bitten by cats ${ }^{4}$; all 3 cat bites were sustained by elderly women in the study. In regards to dog bites, majority of women were house-wives (Table 1). Maximum cases were schoolgoing children <10 yr old in the present study; this could be due to the tendency on part of children to get intimate and play with animals ${ }^{1}$ (Table 1). Commonest site of bite reported in the study was leg followed by face. Additionally, bite on finger, scratches on legs \& thigh also subsequently led to rabies ${ }^{6}$ (Table 4).

No bite was reported by 5 patients; h/o of intimate contact with puppies was present in some of these cases (Table 4, 5). It is possible that rabies can occur if saliva from a rabid animal gets directly into their eyes, nose, mouth or a wound. Scratches, abrasions, open wounds, or mucous membranes contaminated with saliva or other potentially infectious material (such as brain tissue) from a rabid animal constitute non-bite exposures. Occasionally reports of non-bite exposure were such that post exposure prophylaxis was also given ${ }^{7}$.

However, such details are not available in the present study.

Deaths due to rabies in humans and animals are estimated to be the highest in India ${ }^{8}$. It was observed during the study that cases were from different districts spread all over Telangana state including Hyderabad, Ranga reddy, Nizamabad, Mahaboob nagar, Warangal, Medak, Nalgonda, Adilabad, Khammam and Karimnagar and also from Andhra Pradesh ${ }^{4}, 3$ from Karnataka and 2 from Madhya Pradesh. It may be deduced from the observation that these areas indeed could be endemic for the infection.

Out of 91 stray dog bites, there was h/o of the dog inflicting bites on few more persons in the community before getting euthanized. Some of those dogs died of the disease, while some remained apparently healthy and alive. H/o status of dog could not be gathered in all cases. It is known that provoked/unprovoked bites by about $1-5 \%$ of infected animals which do not exhibit any signs of the disease can transmit the infection to humans due to carrier state ${ }^{5}$. There was H/o of bites by pet $\operatorname{dogs}^{9}$ (Table 4 ).

Two bites, one each on hand and face were inflicted by pet dogs with uncertain h/o immunization and the dogs were alive at the time of admission of the patient, 2 months and one month respectively. It needs to be further elucidated whether partial immunization protected the animals from developing the clinical disease while still is able to transmit the infection ${ }^{10}$.

The present study revealed a long range of incubation period from 10 days to 3 yrs (Table no.3) with 45 cases developing disease 1-3 months after the bite. However, there were fulminant cases with deaths occurring in 5-9 days. In 6 cases, death occurred 1-3 yrs after the bite and one case after 25 yrs. A similar case report was published by SK Shankar et al in the year $2012^{11}$.

The reasons for this enormous variability in incubation period are largely unknown. Rabies virus remains cell-free after implantation, allows itself to replicate slowly in muscle cells, may remain latent in macrophages and start invading CNS - weeks, months or years later. Virus attaches to neural axons through lipoprotein receptors, enters the axon and travels at an approximate rate of 1-40 cm/day to reach the CNS and also passes down the axons to the skin and salivary glands. Most of the damage occurs in Hypothalamus and death results due to cardio respiratory arrest ${ }^{12}$.

Clinical suspicion is critical to a timely diagnosis of rabies ${ }^{12}$. Signs and symptoms of encephalopathy, $\mathrm{h} / \mathrm{o}$ animal bite, signs of autonomic disturbance in the absence of coma as were noted in the present study hydrophobia, aerophobia, hyper salivation, hyperventilation, flaccid paralysis, localized paraesthesia-all can be clinical features of rabies (Table no.1).

The commonest presenting feature at the time of admission was hydrophobia (Table no. 1). Commonest site of bite was observed to be leg (44) followed by face (18) (Table 4). All bites were of class lIIsingle/multiple transdermal bites /scratches and licks on broken skin ${ }^{1}$. Five cases with H/o no bite had intimate contact with puppies (Table 4); transmission could be due to infected saliva of rabied puppy/aerosols involving patient's mucous membranes. 30 patients gave history of washing the wound; details of time and method of washing not recorded systematically; while some washed with plain water, others applied antiseptics (Table 5). Two patients gave h/o applying herbal preparations and bandaging the wound tightly (Table 5), which should have been strictly avoided. Measures like bandaging/suturing the dog bite wound will enhance the risk of driving the virus deeper into the wound facilitating its multiplication ${ }^{13}$.

No local treatment was given in rest of the cases (Table 5). The standard recommendations insist on not just washing but scrubbing any bite wound immediately and aggressively with soap and flush thoroughly with water and use antiseptics such as betadine liberally to facilitate removal of viral particles and to certain extent facilitate immobilization of the virus locally ${ }^{3}$.

Unlike most other viral illnesses, infection with rabies does not lead to viremia; hence no antibodies are produced in the patient. This necessitates passive administration of rabies immunoglobulins, human/equine. It was observed in the present study that only 3 rabies patients received RIG; one received 6 days after the bite (Table 5). Another patient with a stray dog bite on leg, not washed wound and not taken RIG, received full course of ARV, developed rabies after one year.

This needs absolute attention. There is abundant evidence in literature to say that optimal post exposure prophylaxis in rabies consists of all three measures-local cleansing, passive antibody administration and active 
immunization as early as possible; rabies had occurred whenever one of these were omitted. Since local viral neutralization is an important means of eliminating virus from the wound, any lapse in this aspect can prove fatal.

It is also very important and absolutely necessary to administer the antibody in the right dose, using the proper technique and into all wounds/scratches as early as possible after the attack. Unlike other viral diseases, infection with rabies virus does not lead to phase of viremia; hence no antibodies are produced. Passive antibody administration in the form of RIG either human/equine may be helpful.

It is also observed that blood levels of RIG are not high after parenteral administration ${ }^{10}$. Hence, WHO recommends local infiltration of the total dose at the site/sites of bite that is anatomically feasible and any leftover RIG should be given parenterally ${ }^{10}$.

RIG should not be given later than 7 days as it may not only be not helpful but counterproductive, since ARV starts building up active immunity, RIG at that stage may lead to immunological interference and antibody elimination ${ }^{12}$.

In the present study, it was observed that ARV was taken by 30 patients (Table 5). 2 of them received 5 doses, of which one patient, a woman aged $60 \mathrm{yrs}$, a house wife with h/o stray dog bites on face, washed the wound and had RIG infiltered within $6 \mathrm{hrs}$ of bite, went on to develop rabies after 1 month. The reason can be attributed to immunosupression in the elderly age compounded by any co morbid condition leading to failure of seroconversion and development of protective titres ${ }^{10}$. This case serves as an eye-opener towards the need of measurement of anti rabies neutralization antibody titres following post exposure prophylaxis.

7 reported having received 4 doses of ARV, 9 received 3 doses and 11 patients reported one dose administration (Table 5). One patient reported receiving 1 dose 20 days after the bite, one day prior to onset of disease. These patients subsequently progressed towards rabies probably because of partial immunization with under protective antibody titer/ no wound management/ no RIG.

All 98 rabies cases died. While one patient died in less than $1 \mathrm{hr}$ of developing the disease, some died in $<24$ hrs - 48 hrs. Most cases died in 3-12 hrs (Table 6).

\section{Conclusions}

Rabies is a condition that needs to be aggressively addressed

$>$ It is not treatable but $100 \%$ vaccine-preventable disease.

$>$ Vaccinating dogs is the most cost-effective strategy for preventing rabies both in animals and humans.

$>$ No animal bite should be taken easy; all mammals are potentially capable of transmitting the infection.

$>$ Effective post exposure prophylaxis includes frequent washing of the wound/wounds with soap and water only, proper local infiltration of RIG and full course of ARV.

$>$ Check for protective post vaccination antibody titres in humans $(>0.5 \mathrm{IU} / \mathrm{ml})$.

$>$ Pet animals to be regularly immunized; children should be cautioned against intimacy.

$>$ Awareness regarding silent rabies and carrier state in dogs.

$>$ Pre-exposure immunization for those spending a lot of time outdoors and those living in areas with a significant risk of exposure to dog bites, for people in certain high-risk occupations such as laboratory workers dealing with live rabies virus and other rabies-related viruses and people involved in any activities that might bring them professionally (veterinary, vaccine production units) into direct contact with rabied mammals including bats and other mammals in rabies-affected areas ${ }^{1}$.

$>$ Elimination of human rabies due to dog bites by 2030 -are we anywhere close?

[1]. Rabies; WHO fact sheet; updated, March 2016

\section{References}

[2]. www.cdc.gov/rabies/Apr 18, 2016

[3]. Understanding Rabies www.humanesociety.org/animals/resources/facts/rabies.html

[4]. $\quad$ Emerg Infect Dis. 2012 Jul; 18(7): 1169. Etymologia: Rabies

[5]. Carrier or Asymptomatic Rabies - Medscape www.medscape.com/viewarticle

[6]. Journal of Applied and Natural Science 6 (1): 38-40 (2014) JANS A study on dog bite incidence in Union Territory of Dadra \& Nagar Haveli, India, V. Khan, D. B. Zala, K. M. Joshi and V.K. Das

[7]. 5year analysis of reported dog bites in Baghdad city: Dr.Maral fathalla thabit, MBCHB, FICM/FM, Al-kindy Col Med J Vol.8; No.2, 2012 P: 151

[8]. CDC-Transmission: Exposure to the Virus - Rabies

[9]. Apr22, 2016, www.cdc.gov/rabies/transmission/exposure.html

[10]. Case Studies-Europa.eu - ec.europa.eu/dgs/health_food-safety/information

[11]. WHO: rabies epidemiology - World Health Organization, www.who.int/rabies/epidemiology

[12]. Rabies viral encephalitis with probable 25 year incubation period! S.K.Shankar,

[13]. Anita Mahadevan, Ann Indian Acad Neurol. 2012 Jul-Sep; 15(3): 221-223

[14]. Oxford Journals, Medicine \& Health, Clinical Infectious Diseases, Volume 30, Issue 1, Pp. 4-12

[15]. Rabies: Manual for doctors; Prof M.K.Sudarshan; July 1999; pg No 15 